\title{
Management of Supernumerary Teeth: Clinicians Enigma
}

\author{
Bhatnagar P*, Chaudhary S, Manuja N and Kaur \\ H \\ Department of Pedodontics and Preventive Dentistry, \\ Kothiwal Dental College and Research Centre, India \\ *Corresponding author: Bhatnagar P, Department of \\ Pedodontics and Preventive Dentistry, Kothiwal Dental \\ College and Research Centre, Moradabad, India
}

Received: May 17, 2017; Accepted: June 15, 2017; Published: J une 23, 2017

\begin{abstract}
Supernumerary tooth (ST) is a developmental anomaly that has been argued to arise from multiple aetiologies. These may occur in primary and permanent dentition. They are classified based on form, morphology, location, and occurrence. However, combinations of environmental and genetic factors are the most probable cause for the same. Supernumerary teeth cause a range of complications like crowding, displacement, dilacerations, cyst formation, and so forth. The present case deals with the problem of minor crowding that arose due to the presence of supernumerary teeth and its management.
\end{abstract}

Keywords: Supernumerary tooth; Extraction; Crowding; Orthodontic intervention

\section{Introduction}

Supernumerary tooth (ST) is defined as "any tooth or odontogenic structure that is formed from tooth germ in excess of usual number for any given region of the dental arch [1,2]. They may be unilateral or bilateral and single or multiple, in distribution, occur in any part of the tooth bearing areas in both dental arches, and may occur in primary and permanentdentition [3]. These ST could occur at any region of the dental arch and most commonly in premaxilla. There are several hypotheses which have been proposed to explain the occurrence of ST [2,4]. A combination of environmental and genetic factors is most popular causes [5]. Supernumerary teeth cause a range of complications varying from crowding to cyst formation. However, the position of ST is buccal or lingual or within the arch. Localization of ST plays a major role in diagnosis and treatment, especially if surgical intervention is needed [6]. Though, it is clear that early treatment can possibly prevent further complications, some authors anecdotally suggested that this approach is hazardous due to possible risk of damage to the developing tooth germs. Location of ST must be established by different imaging techniques. Although, combinations of intraoral radiographs with panoramic radiographs are usually able to provide the required information, these procedures do not always provide exact details concerning the 3 -dimensional (3D) relationship of the ST $[2,6,7]$.The below mentioned case report highlights the early diagnosis and prompt management of supernumerary teeth.

\section{Case Presentation}

A 13-year-old girl reported to the Department of Pedodontics and Preventive Dentistry; KDCRC Moradabad, with the chief complaint of two teeth present behind the upper two front teeth. On intraoral examination, two supernumerary teeth were seen between permanent maxillary central incisors and Ellis class IV fracture in relation to 11 (Figure 1A,B and C). Minor crowding of the maxillary arch was also seen. Dentition stage was permanent with Angle's class I molar relationship bilaterally.

\section{Treatment plan}

It was decided to extract the two supernumerary teeth followed by root canal treatment and prosthetic rehabilitation of 11. Orthodontic intervention was planned for correction of minor crowding in maxillary arch.

\section{Treatment done}

The supernumerary teeth were extracted under local anesthesia (Figure 2A,B and C). One week later, root canal treatment was done in relation to 11. After hiatus of one week, fibre post cementation and core build up was done sequenced by acrylic crown on 11 (Figure $3 \mathrm{~A}, \mathrm{~B}, \mathrm{C}$, and $\mathrm{D})$.

For the alignment of the crowded maxillary arch (Figure 4A), permanent maxillary first molars were banded and complete maxillary arch bonding was done by MBT (Figure 4B). A $0.016 \times 0.022$ inch $\mathrm{NiTi}$ wire followed by $0.016 \times 0.022$ inch stainless steel wire was placed in the maxillary arch in due course of time. Slight proximal stripping was done in relation to mesial aspect of 21 . The patient was called for monthly follow-up. Six months later minor crowding was aligned. De-bonding was done and the patient was given a fixed retainer (Figure 4C).

\section{Discussion}

Supernumerary teeth are tooth or tooth like structures in excess of the usual configuration of the normal number of deciduous or permanent teeth. Although a lot of theories have been proposed to explain the development of supernumerary teeth, yet the exact aetiology remains obscure [8]. The most accepted theory is the hyperactivity theory which proposes that supernumerary teeth are formed as a result of localized and independent hyperactivity of dental lamina [9]. ST classified based on morphology, location, position, orientation (Figure 5).

It is essential to identify the presence of ST clinically and radio graphically formulating a definite treatment plan. Clinical complications such as midline diastema, displacement, delayed or failure of eruption, rotations, and impaction of teeth might leave a way of identification of ST. Identification and localization of ST are essential for the management if needed [1].

Several authors have given different opinions regarding the extraction timing for the management of ST. The treatment options for managing ST depend on their orientation and position, the age of the patient, and any associated complications. Similarly, few
Austin J Dent - Volume 4 Issue 5 - 2017

ISSN : 2381-9189 | www.austinpublishing group.com

Bhatnagar et al. () All rights are reserved 


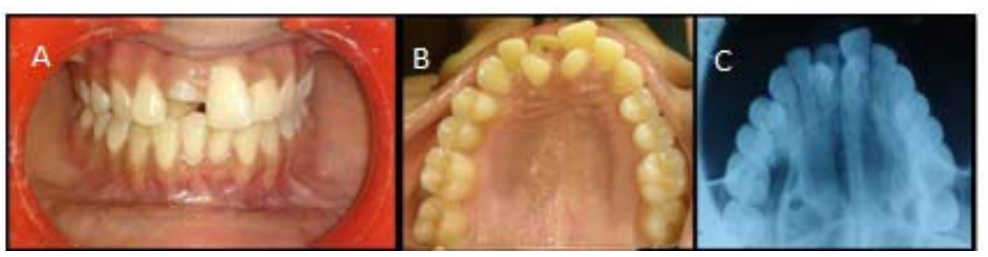

Figure 1: Pre operative (a) Frontal view (b) Maxillary occlusal view (c) Occlusal radiograph.

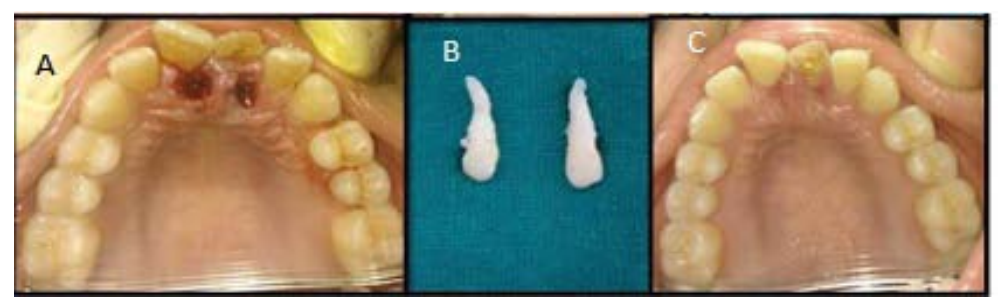

Figure 2: Post operative (a) Post operative occlusal after extraction (b) Extracted supernumerary teeth (c) Post operative after one week.

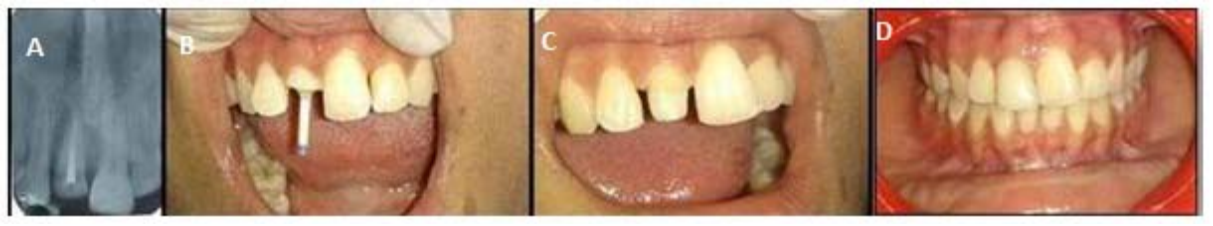

Figure 3: Endodontic treatment and Prosthetic Rehabilitation (a) Obturation (b) Fibre post and core (c) Crown cutting (d) Crown cementation.

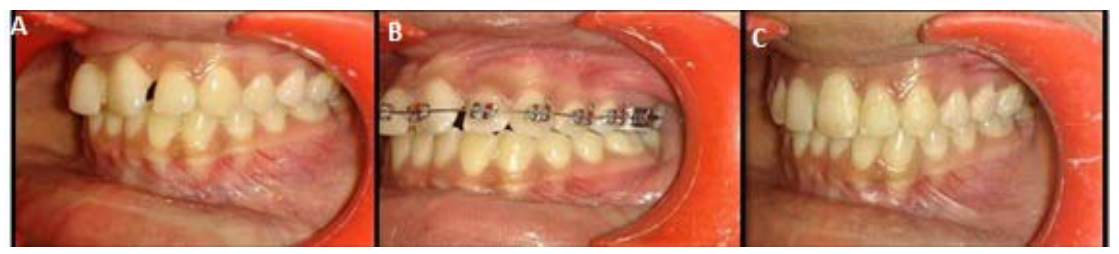

Figure 4: Orthodontic Therapy (a) Pre operative (b) After MBT bracket placement (Intermediate) (c) Post operative after de-bonding.

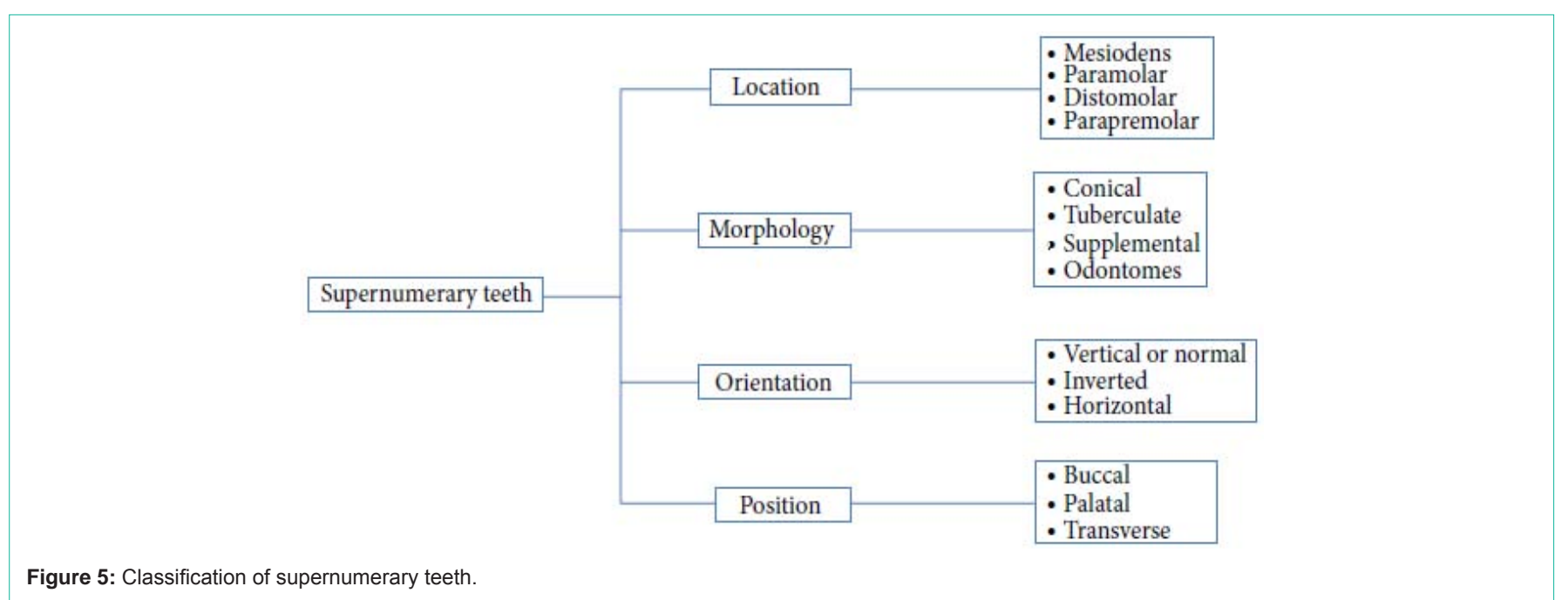

authors reported that early identification and removal of ST, in contrast to some authors which may suggest abrupt removal of ST, is not essential if there was no associated pathology. Removal of ST is not always a treatment of choice; they may be reviewed if the tooth is not creating any problem. Hogstrom and Andersson suggested two different opinions where the ST should be removed as early as upon identification or should wait until complete root formation of adjacent teeth. The optimal time for surgical intervention, however, 
remains contentious. It is essential to remove ST at a young age if it is damaging adjacent teeth or causing any other complication. Recently, Omer and colleagues reported based on a retrospective analysis the ideal age of removal of ST 6 to 7 years. The majority of delayed permanent incisors erupt spontaneously if sufficient space is created at the time of removal of the ST and if postoperative space is maintained. Permanent maxillary incisor teeth still remained unerupted with near complete apical formation; orthodontic bracket and chain may be used to facilitate orthodontic traction while in some cases based on angulations of impacted teeth caused by ST, orthodontic bracket and chain placement at the time of surgical removal ST may be essential for the eruption [1].

The presence case also dealt with the malalignment due to the presence of supernumerary teeth on the palatal aspect of permanent maxillary central incisors. The crowding was due to the presence of an extra tooth that might have led to disruption in the normal alignment of the maxillary anteriors. Therefore, extraction of the supernumeraries followed by orthodontic correction was opted as the best possible treatment. The crowding was resolved after six months of orthodontic therapy. For the retentive phase, fixed retainer was given to the patient for a long period of time to prevent relapse [10].

\section{References}

1. Mallineni KS. Supernumerary Teeth: Review of the Literature with Recent Updates. Conference Papers in Science. 2014; 1-6.
2. Omer RS, Anthonappa RP, King NM. Determination of the optimum time for surgical removal of unerupted anterior supernumerary teeth. Pediatric Dent. 2010; 32: 14-20.

3. King NM, Lee AM, Wan PK. Multiple supernumerary premolars: their occurrence in three patients. Aust Dent J. 1993; 38: 11-16.

4. Anthonappa RP, Omer RSM, King NM. Characteristics of 283 supernumerary teeth in Southern Chinese children. Oral Surg Oral Med Oral Pathol Oral Radiol Endod. 2008; 105: 48-54.

5. Brook H. A unifying aetiological explanation for anomalies of human tooth number and size Arch Oral Biol. 1984; 29: 373-378.

6. Mallineni SK. Radiographic localization of supernumerary teeth in the maxilla. University of Hong Kong. 2011.

7. Anthonappa RP, King NM, Rabie ABM, Mallineni SK. Reliability of panoramic radiographs for identifying super numerary teeth in children. Int $\mathrm{J}$ Paediatr Dent. 2012; 22: 37-43.

8. Pele E, MelnikBlaszczak M, Szyszkowska AM. Rare cases of supernumerary teeth. Ann Uni Mar Cur. 2006: 849-853.

9. Mehta $\vee$, Ahmad AS. Surgical Management of a Supplemental Permanent Maxillary Tooth with Mesiodens in a Non-Syndromic Patient: A Case Report. J Dent Health Oral Disord Ther. 2017; 6: 1-4.

10. Revathi M, Patil A, Hemavatiwalikar, Agarwal K. Surgical and Orthodontic Management of Mesiodens and Dilacerated Supernumerary Tooth: A case report. Surgical and orthodontic management of supernumerary tooth. 2016; 2: 96-99.
Austin J Dent - Volume 4 Issue 5 - 2017

ISSN : 2381-9189 | www.austinpublishing group.com

Bhatnagar et al. (C) All rights are reserved
Citation: Bhatnagar P, Chaudhary S, Manuja N and Kaur H. Management of Supernumerary Teeth: Clinicians Enigma. Austin J Dent. 2017; 4(5): 1084 